\title{
Innovación en gestión de recursos humanos: la relevancia de las redes inter-organizativas de intercambio de conocimiento
}

\author{
Manuel Septiem ${ }^{1 *}$, Carlos Martin-Rios ${ }^{2}$
}

Resumen: El presente artículo utiliza metodología de redes sociales para analizar empíricamente el posible impacto positivo de la participación en redes de intercambio de conocimiento para la implementación de innovaciones en gestión de GRH. Los resultados indican que aquellas empresas que participan activamente en el establecimiento de relaciones de intercambio de conocimiento de GRH con otras empresas, tanto a través de acuerdos formales-contractuales como mediante relaciones informales-colaborativas, tienen una propensión mayor a realizar innovaciones en esta área. Como resultado, las empresas más expuestas a nuevos conocimientos son en mayor medida más innovadoras en sus sistemas de gestión de GRH. Se concluye que el establecimiento de relaciones inter-organizativas tiene efectos positivos en la introducción de prácticas innovadoras de gestión de GRH.

Palabras clave: Innovación; redes sociales; gestión de recursos humanos; redes inter-organizacionales; intercambio de conocimiento; innovación en gestión de personas; relaciones colaborativas.

\begin{abstract}
Title: Innovative human resource management: the salience of inter-firm knowledge networks.
This work draws on social network analysis to empirically assess the relationship between firm participation in inter-firm knowledge networks and innovation in human resource management (HRM). Results suggest that participation in the establishment of inter-firm knowledge exchanges, both through formal and informal networks and relationships, has a positive relationship with the implementation of HRM innovations. Findings suggest that firms that actively participate in inter-firm knowledge networks obtain knowledge resources and tend to be more innovative in the HRM system and practices.
\end{abstract}

Keywords: Innovation; social networks; human resource management (HRM); inter-firm networks; knowledge networks; knowledge exchange; innovation in people management; collaborative relationships.

Submitted: May $2^{\text {nd }} 2017 /$ Approved: September $8^{\text {th }} 2017$

\section{Introducción}

Para las empresas que tratan de ser competitivas en un entorno económico de creciente dinamismo y complejidad las posibilidades de supervivencia residen, con frecuencia, en la capacidad para movilizar, integrar y administrar múltiples recursos con el fin de afrontar la incertidumbre y las exigencias propias de las estrategias innovadoras (Martin-Rios y Pasamar, 2017). Uno de los recursos críticos que requiere ser gestionado eficazmente son las personas que conforman la organización (Huselid, 1995). En concreto, la literatura ha prestado especial atención al desarrollo de prácticas de gestión de recursos humanos (GRH en adelante) altamente innovadoras con las que atraer, motivar y retener satisfactoriamente a las personas claves de la organización (Martin-Rios, 2014; Martin-Rios y Erhardt, 2016; Martin-Rios, Pougnet y Nogareda, 2017). Dicha literatura ha constatado que una GRH eficaz constituye una fuente potencial de ventaja competitiva sostenida para las empresas (Lepak y Snell, 2002). Buena parte de estos estudios fundamentan sus hallazgos en los principios de la teoría de los recursos y las capacidades para hacer hincapié en la naturaleza única de cada organización y en la explotación de sus diferencias con otras empresas para obtener ventajas competitivas sostenibles (Barney, 1991; Dierickx y Cool, 1989; Martin-Rios y Erhardt, 2017; Wright, Dunford y Snell, 2001). De tal forma que la apropiación de conocimiento en GRH y su puesta en práctica para gestionar de forma efectiva el capital humano constituye un recurso estratégico que contribuye a innovar en GRH para diferenciarse y aventajar a la competencia (Martin-Rios, 2014; Paauwe y Boselie, 2005).

Curiosamente, a pesar de la extrema relevancia que tiene la obtención de conocimiento de GRH en la posterior implementación de innovaciones en GRH, la literatura se ha mostrado reticente a analizar en profundidad cuáles son los canales a través de los que se intercambia dicho conocimiento y qué efectos tiene la participación en dichos intercambios en las dinámicas innovadoras en GRH de las empresas (Martin-Rios, 2014). Las lagunas en la literatura académica presentan oportunidades de avanzar nuestro conocimiento. En concreto, el presente estudio hace frente a dos cuestiones centrales: ¿qué características y propiedades estructurales poseen las redes inter-organizacionales para el intercambio de conocimiento de GRH? Asimismo, ¿cuál es la relación entre la participación en la red -en términos de posibilidades de intercambio de conocimiento de GRH-y la implementación de innovaciones en este campo?

(1) Universitat de les Illes Balears. Departamento de Economía de la Empresa, España.

(2) Ecole hôtelière de Lausanne // HES-SO University of Applied Sciences Western Switzerland, Suiza.

*Autor de correspondencia: m.septiem@uib.eu 
La atención del estudio se centra en la importancia de las características y propiedades estructurales de las relaciones formales y las relaciones de colaboración como factores que la literatura de redes interorganizativas señala que tienen un mayor impacto en el intercambio de conocimiento (Martin-Rios y Septiem, 2013; Septiem y MartinRios, 2011). Este análisis es especialmente pertinente a la hora de analizar los aspectos estructurales del intercambio de conocimiento (tales como la intensidad y la frecuencia de las relaciones) que relacionan la participación en redes de conocimiento y la implementación de innovaciones (por ejemplo, la posición de centralidad en la red).

La organización del artículo es la siguiente: en primer lugar, se revisa la literatura relativa al intercambio de conocimiento entre empresas según los diversos modelos de relaciones existentes. A continuación, desde la teoría de las redes sociales se analizan las características y propiedades estructurales que influyen en el establecimiento de intercambios de conocimiento, específicamente los intercambios de conocimiento en GRH. A continuación se explicitan los modelos de investigación utilizados. En el apartado de resultados se analiza la existencia de relaciones y el grado de participación de las empresas que participan en redes de intercambio de conocimiento de GRH y los mecanismos estructurales e instrumentales que subyacen a estas redes. Concluimos con un conjunto de implicaciones para la práctica y la investigación.

\section{Revisión de la literatura}

\section{Teoría organizacional de la red social y las relaciones inter-orga- nizacionales}

La teoría de las redes sociales ofrece razones teóricas por las cuales las empresas pueden participar activamente en el intercambio de conocimiento de GRH entre organizaciones. Dentro de la investigación organizacional de la red social (Granovetter, 1979; Kilduff y Brass, 2010; Rubinstein et al., 2016), los investigadores han examinado el papel de las redes para sugerir que el establecimiento y la participación en relaciones inter-organizacionales ofrecen beneficios a las empresas en forma de circulación de conocimiento (Martin-Rios y Erhardt, 2017; Martin-Rios y Septiem, 2013). Recientemente, dentro de la literatura de gestión empresarial, los investigadores han expresado su interés en las redes de intercambio de conocimiento como una prioridad para aquellas empresas cuyo objetivo es participar en los flujos de conocimiento (Parmigiani y Rivera-Santos, 2011). Estos estudios en general, destacan el papel de los factores económicos y sociales en el impulso de la participación de la empresa, partiendo de trabajos anteriores que hacían hincapié en los factores institucionales relativos a las presiones externas y las presiones de la conformidad normativa (Fligstein, 1985; Westphal y Zajac, 1997) ${ }^{1}$.

En lo que respecta al análisis de las redes de intercambio de conocimiento, la investigación se ha centrado en dirimir la relevancia de las relaciones tanto formales como informales entre organizaciones, que favorecen el intercambio y la prestación conjunta de conocimiento sobre diversos aspectos relativos al ámbito tecnológico, productivo (productos y/o servicios) y de gestión organizativo (Powell y Grodal, 2005; Sammarra y Biggiero, 2008). Específicamente, estos estudios examinan el impacto que dichas relaciones tienen en diversos resultados empresariales, tales como el aprendizaje organizacional y la innovación (Alexander y Childe, 2013). En general, se considera que las redes inter-empresariales y el intercambio del conocimiento que se produce en ellas es una práctica arraigada (Uzzi 1996), que favorece unos niveles considerables de intercambio (Powell, Koput, y SmithDoerr, 1996), en base a relaciones descentralizadas (focos de poder repartidos) (Sammarra y Biggiero, 2008) y niveles significativos de reciprocidad (Easterby-Smith et al., 2008). Dichas redes adquieren, con frecuencia, carácter formal (contractual o estratégico), son altamente selectivas y centralizadas. Es decir, un número limitado de empresas ocupan las posiciones centrales (por ejemplo, grandes multinacionales como el grupo Toyota) y definen las posibilidades de acceso al resto de empresas (en el ejemplo, distribuidores, suministradores y clientes de Toyota) (Dyer y Nobeoka, 2000). Más recientemente, la literatura ha revelado la existencia de redes informales o colaborativas entre empresas (Cross, Nohria y Parker, 2012). Estas relaciones carecen a priori de carácter comercial y, por ende, tanto el acceso como la posición que ocupan los diversos actores es menos selectivo que en las redes formales.

Un argumento recurrente sugiere que la proximidad física y cognitiva entre empresas e instituciones favorece un mejor acceso a los flujos de conocimiento y representa una mejora en la capacidad innovadora de estas empresas (Uzzi, 1996). Sin embargo, Breschi y Lissoni (2001) sugieren la necesidad de nuevas investigaciones orientadas a mejorar nuestra comprensión del intercambio de conocimientos localizados, cuestionando que la información y el conocimiento fluyan sin restricciones en las redes locales. Particularmente importantes son las nuevas herramientas metodológicas en la medición de la difusión del conocimiento a través del Análisis de Redes Sociales (ARS) (Borgatti, 2007), que han permitido una mejor comprensión de los elementos generadores de redes de conocimiento.

\section{Las relaciones inter-organizacionales de intercambio de cono- cimiento en GRH}

Uno de los escenarios más apropiados para la difusión y adquisición de conocimiento es a través de la red social, definida como el patrón de comunicación, amistad, asesoramiento o apoyo que existe entre los miembros de un sistema social (Burt \& Minor, 1983). Las redes sociales, sus características y estructura son cruciales para determinar los flujos de difusión de ideas, innovaciones o experiencias (Burt \& Minor 1983; Scott 1991; Wellman 1983). Al utilizar el concepto de red social hacemos referencia a la teoría de redes y el análisis estructural o de redes sociales según la cual las personas se encuentran conectadas unas con otras formando canales de comunicación e influencia (Granovetter, 1985) a través de los que tienen lugar también las relaciones inter-organizacionales. Por ello la teoría de redes sociales se utiliza ampliamente como marco conceptual para el estudio del intercambio de ideas y conocimiento en una amplia variedad de contextos, incluido el inter-organizacional.

(1) Por ejemplo, Fligstein (1985) analizó la propagación de formas multidivisionales y Burns y Wholey (1993) exploraron la adopción de estructuras matriciales. Otros autores han analizado el intercambio de conocimientos en relación a los procesos del bloqueo de intercambio de una red (e.g. Westphal y Zajac, 1997) o la adopción de innovaciones en el campo de la medicina (e.g. Coleman, Katz y Menzel, 1966; Becker, 1970). 
Sin embargo, buena parte de la literatura de GRH ha centrado su atención en analizar los flujos de conocimiento de GRH en el nivel intra-organizativo. Por ejemplo, bajo el enfoque de la dirección internacional de recursos humanos, diversos estudios han analizado la transferencia de conocimiento de prácticas específicas de GRH (tales como la evaluación del desempeño o el sistema de establecimiento de incentivos) desde la sede central de la multinacional a sus diversas subsidiarias (Björkman y Lervik, 2007; Martin-Rios y Erhardt, 2008), o entre organizaciones participantes en joint ventures internacionales y alianzas estratégicas (Schuler, Jackson, y Luo, 2003).

En base a la evidencia empírica señalada en el anterior apartado, cabría esperar un mayor número de estudios sobre la existencia e importancia de la trasmisión de conocimiento de GRH entre empresas independientes. Sin embargo, la literatura sobre dichos intercambios es limitada como diversos estudios ponen de manifiesto (Brass, 1995; Legnick-Hall y Legnick-Hall, 2003; Martin-Rios, 2014). La mayor parte del trabajo realizado hasta la fecha se ha enfocado en analizar la difusión de determinadas prácticas de RH. Por ejemplo, el trabajo de Williamson y Cable (2003) se centra en los procesos de selección de los equipos de alta dirección para sugerir que el contexto social en el que las empresas están imbricadas determina la toma de decisiones en temas de GRH, tales como las decisiones de contratación entre las empresas Fortune 500). Asimismo, Mazza y Alvarez (2000) analizan la función de los periódicos y la de prensa popular en la difusión de conocimiento de GRH. Dichos estudios ponen de manifiesto la relación positiva entre el establecimiento de relaciones contractuales entre empresas y el intercambio conocimiento de GRH (Martin-Rios y Erhardt, 2008; Schuler, Jackson, y Luo, 2003). Cabe esperar que dichos intercambios tengan lugar asimismo cuando las relaciones se establecen de manera informal y cooperativa. Sin embargo, la falta de estudios no permite respaldar estas afirmaciones. Asimismo, hay una carencia de estudios sobre el efecto que el intercambio de conocimiento en GRH entre empresas independientes tiene en el esfuerzo innovador en GRH por parte de las empresas involucradas.

El presente estudio contribuye a solventar parte de estas carencias mediante un análisis empírico de las relaciones de intercambio de conocimiento de GRH entre empresas independientes ubicadas en un parque científico y tecnológico. Para analizar todas estas cuestiones nos basamos en un enfoque multidisciplinar que integra las ideas de la investigación sobre los flujos de conocimiento entre organizaciones (Easterby-Smith, Lyles, y Tsang, 2008; Powell, Koput y Smith-Doerr, 1996) y la teoría de redes sociales (Granovetter, 1985), que nos aportará una visión desde la perspectiva de la red social para analizar empíricamente los factores mencionados en los procesos de intercambio.

\section{Contexto y Metodología}

\section{Los parques científicos y tecnológicos}

Los grupos de empresas de ciencia y tecnología conocidos como "parques" surgieron en los EE.UU. en la década de 1950, en un intento por aumentar la productividad de empresas de base tecnológica por la proximidad física o la aglomeración territorial (Castells y Hall, 1994) de dichas empresas con las administraciones públicas, los centros de I+D y las universidades. Tras su éxito en los EE.UU., dichos clusters se extendieron a Europa en los años 60 y 70 (por ejemplo, Cambridge en Reino Unido, Sophia-Antipolis en Francia, o Medicon Valley en Dinamarca-Suecia) y al sur de Asia en los 80 y 90 (por ejemplo, $A^{\star}$ START en Singapur).

La lógica detrás de estos parques tecnológicos fue que la proximidad de la industria tecnológica puede ayudar en la formación de vínculos de red y facilitar contactos entre empresas y, en particular, favorecer las interacciones interpersonales a través de las cuales el conocimiento se espera que sea intercambiado (Diez-Vial y Montoro-Sanchez, 2016; Sammarra y Biggiero, 2008).

El parque de ciencia y tecnología que hemos seleccionado para nuestra investigación se encuentra en el área metropolitana de Madrid (España). En el año 2000, una universidad pública junto con organismos públicos locales, regionales y nacionales, puso en marcha el parque. Hoy día constituye un ejemplo de parque científico y tecnológico donde la universidad ha sabido combinar una incubadora de empresas y el apoyo institucional a la alta tecnología de reciente creación (start-ups), junto con los servicios básicos a las empresas ya consolidadas en el mercado (algunas de ellas, con varios años de antigüedad). La universidad a través de su oficina de apoyo a empresas oportunidades para el desarrollo de flujos de conocimiento y el fomento de la innovación. Como tal, desempeña un papel preponderante en la creación y desarrollo de las relaciones entre empresas.

El parque se compone de 38 empresas (la mayoría de ellos miembros de la asociación local de empresarios ${ }^{2}$ ), incluyendo las start-ups, las empresas establecidas, y las subsidiarias de empresas multinacionales. La incubadora de empresas incluye proyectos empresariales e ideas (cinco en total) y 12 spin-offs universitarios y de desarrollo de proyectos empresariales. Por último, hay seis centros de I+D financiados con fondos públicos, incluidos dos institutos de investigación y consorcios de $\mathrm{I}+\mathrm{D}$ entre empresas y la universidad.

\section{Recogida de datos}

El análisis de las redes sociales estudia, tanto las redes completas, es decir, todos los vínculos que contienen las relaciones específicas dentro de una población definida, como las redes personales (también conocidas como redes egocéntricas), donde los lazos estudiados son los que desarrollan las personas individuales (comunidades personales). Para obtener una mejor comprensión del intercambio de conocimientos de GRH entre empresas se optó en este estudio por una red completa de empresas (todas las empresas pertenecientes al PCT). Si bien cada empresa puede $y$, a menudo mantiene, relaciones de intercambio con actores fuera del parque (otras empresas, organismos e instituciones), por cuestiones de proximidad y con el fin de analizar una única red de dimensiones manejables, el presente análisis se centra en los intercambios que cada empresa ubicada en el parque mantiene con el resto de empresas también ubicadas en el parque.

(2) La asociación de empresas del PCT, nace por iniciativa de las propias empresas, en principio para solucionar problemas surgidos en la llegada al parque. Como algún entrevistado comentó: "al principio tratábamos temas como de 'comunidad de vecinos', pero también nos sirvió para conocernos y establecer relaciones comerciales...”. 
Hemos elaborado un cuestionario para trazar el intercambio de conocimiento entre empresas, a través de las personas con responsabilidades para el mantenimiento de relaciones con otras empresas (por ejemplo, directores generales y directores de GHR). El cuestionario sociométrico se basa en las líneas directrices aceptadas en la literatura especializada (en particular, Borgatti, Everett y Freeman 2002; Scott, 2001). La encuesta incluye un listado completo de todas las empresas del PCT, que comprende un total de 51, con exclusión en la muestra de todos los proyectos de negocios en la incubadora de empresas y todos los centros de I+D sin una visión clara del negocio. Este método de lista se ha demostrado fiable en otras investigaciones pues facilita al entrevistado recordar los patrones típicos de interacción (Diez-Vial y Montoro-Sanchez, 2016; Kumar, Stern y Anderson, 1993).

El trabajo de campo duró ocho semanas. Incluyendo la Oficina de Tecnología del PCT Leganés, nuestra muestra estuvo conformada por 51 empresas. Se localizó telefónicamente a cada gerente, de forma que cada empresa tuvo una probabilidad de participar en el estudio del
$100 \%$. Finalmente, se administró el cuestionario a 41 empresas, lo que representa una tasa de respuesta del $80,4 \%$. Las otras 10 empresas declinaron la participación tras varias llamadas telefónicas o, en algunos casos, no pudieron ser contactados. Las entrevistas personales se realizaron con un director ejecutivo o alto directivo por empresa. El método de informante único se ha considerado adecuado tanto en la investigación de GRH como en la de redes sociales (Borgatti y Cross, 2003; Huselid, 1995).

La Tabla 1 presenta los datos descriptivos de la muestra. La edad promedio de las empresas es de 17 años, siendo de 85 años la más antigua de las empresas. El noventa por ciento de las empresas son pequeñas y medianas empresas (PYMEs), mientras que el 10 por ciento son grandes empresas con más de 200 empleados y unas ventas superiores a 15 millones de euros. De acuerdo a la clasificación CNAE-2009, veintisiete de las empresas entrevistadas (53\%) trabajan en servicios de alta tecnología y trece $(25,5 \%)$ son empresas manufactureras de alta o media-alta tecnología.

Tabla 1. Descriptivos

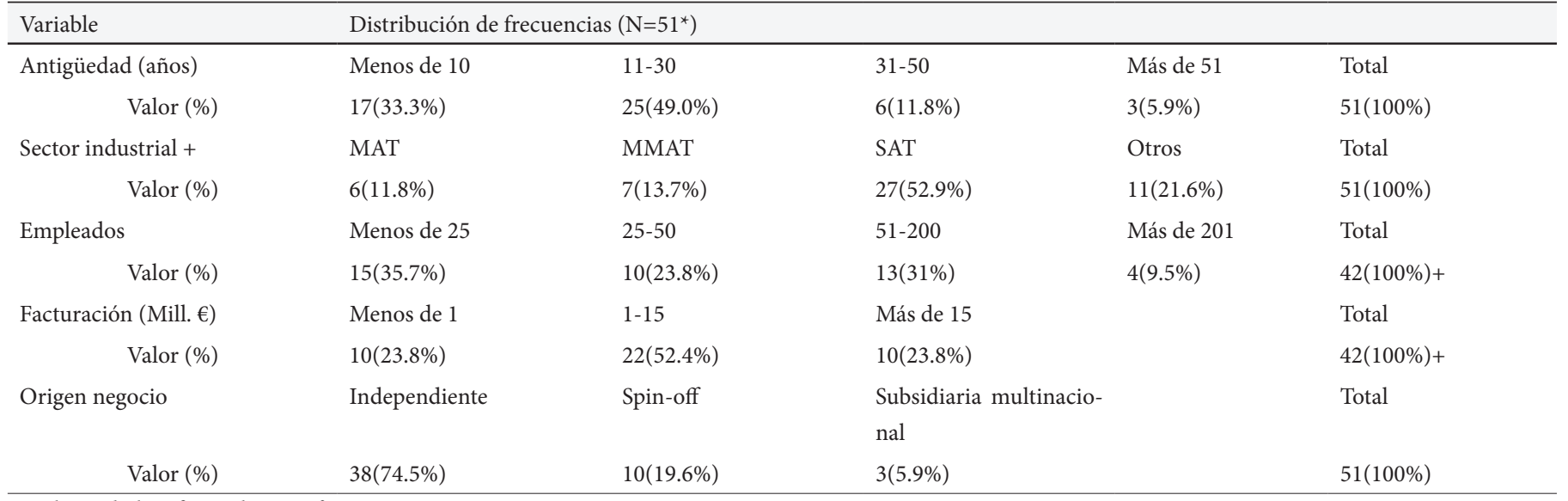

* Incluyendo la Oficina de Transferencia

+ MAT: manufactura alta tecnología; MMAT: manufactura media-alta tecnología; SAT: servicios alta tecnología +9 casos perdidos

! 1 caso perdido

A los encuestados se les pidió hablar de esas organizaciones con los que había intercambiado información de GRH en los últimos seis meses. Los encuestados tuvieron que elegir sus respuestas de una lista que contiene todas las empresas (50) del Parque. No había ninguna restricción sobre el número máximo de selecciones que cada encuestado podía hacer. Clasificamos las relaciones dentro de la red real entre los actores $\mathrm{i}, \mathrm{j}$, $\mathrm{k}$ en formal (transaccional) e informal (personal) o de asesoramiento o relacionada con el grado de confianza (relacional) (Friedman y Podolny, 1992). A los encuestados se les dio ejemplos del tipo de conocimiento para clasificar el tipo de intercambio de información de gestión de GRH. Se les suministró un listado de prácticas de gestión de personas con 10 diferentes prácticas (entre ellas, reclutamiento, selección, formación, compensación y evaluación del desempeño), que los investigadores han utilizado con frecuencia en el campo estratégico de GRH (Huselid, 1995).
Junto al cuestionario de redes sociales, los entrevistados completaron un cuestionario con una serie de preguntas relativas a la GRH en la propia empresa. En una de ellas se solicitó que señalasen cuáles de las prácticas de GRH del listado habían sido introducidas o substancialmente mejoradas en los dos últimos años (con anterioridad a la realización de este estudio) Asimismo, se preguntó por los motivos por los que dichas prácticas de GRH fueron puestas en marcha. De esta forma se puede investigar la relación entre la participación en intercambio de conocimiento e innovación en prácticas de GRH.

\section{Análisis de la red}

Los datos de la red investigada fueron procesados utilizando el paquete de software UCINET (Borgatti et al., 2002). Los mapas de red o "sociogramas" que se presentan a continuación se desarrollaron mediante la NetDraw (Borgatti, 2007). Los sociogramas representan la red como 
un conjunto de nodos que representan a las empresas y un conjunto de líneas dirigidas (las líneas que unen los nodos) (Moreno, 1934) que indican la presencia del intercambio de conocimientos entre los empresas. En la red analizada, los lazos tienen puntas de flecha que indican la dirección de la colaboración. La posición central de cada firma (es decir, el número de lazos que una empresa tiene con otras empresas) está representado por el tamaño del nodo (es decir, mayores nodos representan mayores centralidades) y el valor de los lazos (es decir, la fuerza de la relación) puede ser observado por su espesor (los valores más altos están representados por una línea más gruesa). Los paquetes de software, como los utilizados en esta investigación, de forma automática transforman los datos estadísticos de red para generar sociogramas.

Los datos de la red se introdujeron en una matriz para calcular los índices de la red, que describen la cohesión de toda la red, lo que indica la presencia de las relaciones de red entre las empresas participantes (Reagan y McEvily, 2003) y también la probabilidad de participación en los diversos flujos de conocimiento (Scott, 1991).

\section{Estructura de la red}

Las propiedades estructurales y relacionales definen los aspectos de la configuración de la red y proporcionan información sobre la estructura de las relaciones sociales de estas redes. El análisis estructural examina las relaciones entre las unidades de análisis en lugar de las propiedades individuales de las unidades (Coleman, 1958) para examinar cómo las redes de relaciones se originan. La centralización de la red es una medida a nivel de red que cuantifica el grado jerárquico de la red (Freeman, 1979). Para este trabajo se calcularon tres medidas: centralización, densidad y reciprocidad de las relaciones.

La centralización se calcula mediante la adición de la suma de las diferencias en la centralidad del actor más central a todos los demás y se normaliza dividiendo por el máximo posible de todos los grafos conectados (Borgatti y Everett, 1997). Cuando la medida es grande (valor máximo de 1), significa que los agentes centrales son muy pocos y que los agentes restantes ocupan posiciones mucho menos centrales en la red. Por el contrario, si la centralización de la red es baja (un valor mínimo de 0), significa que la red está poblada por actores que ocupan posiciones centrales de manera similar. También se calculó la media de la centralidad de grado, que se define como un valor medio global de la cantidad de vínculos que tiene un nodo, ya sea de salida (grado de salida) o de entrada (grado entrante) y mide el nivel de participación de una empresa en una red.

La densidad estructural analiza el número de enlaces entre los miembros de la red con respecto al número de enlaces posibles y se registra como un porcentaje (Scott, 1991). La densidad es una indicación de cómo unida es una red y la fuerza de esas relaciones (Wasserman y Faust, 1994). Los valores de densidad varían desde 0 a 1 . El valor calculado para cada red de conocimiento permite interpretar la densidad de los flujos de conocimiento entre las empresas como un índice de la probabilidad de que las empresas intercambien sus conocimientos. También se calculó la suma total del conocimiento compartido o la suma de los grados, que describen la cantidad total de las relaciones a través de las cuales el conocimiento se espera que sea compartido.
La reciprocidad se define como la relación del número de lazos que son recíprocos con el número total de los lazos (Wasserman y Faust 1994), donde $\mathrm{M}$ es el número de parejas completas mutuas y A las díadas asimétricas en la red. Las puntuaciones de esta medida son las proporciones que van desde 0 a 1 . La reciprocidad, por tanto, es referida para determinar la fuerza del vínculo (Granovetter, 1979) o la fuerza de la relación informal entre las empresas, que mide la frecuencia con que las empresas intercambian recíprocamente con respecto al número de intercambios e indica el flujo unidireccional de una empresa a otra. Los enlaces recíprocos se postulan para indicar que lazos son más fuertes que los enlaces unidireccionales. La reciprocidad es un indicador adecuado del grado de reciprocidad y el intercambio recíproco en una red, que se refiere a la confianza, la cohesión social y la credibilidad.

\section{Intercambio de conocimiento e innovación}

De acuerdo con la literatura inter-organizacional, el establecimiento de relaciones y la participación en intercambios entre empresas aumenta la capacidad innovadora y la probabilidad de que las empresas lleven a cabo iniciativas de innovación (Appleyard, 1996; Powell y Grodal, 2005; Sammarra y Biggiero, 2008). Puesto que, hasta la fecha, no se han llevado a cabo estudios que analicen la participación en redes y la actividad innovadora en el ámbito de $\mathrm{GRH}$, en este estudio analizamos dicha relación mediante un análisis por el que segregamos las empresas en dos grandes categorías: por un lado, las empresas que participan en intercambios de GRH a través de redes formales, contractuales (intercambio formal) y en segundo lugar, las empresas que participan en redes de intercambio colaborativo o informales (intercambio informal). Entonces, operacionalizando el tipo de intercambio construimos una matriz de 50x50, donde la codificación de cada empresa como " 1 " si ambas organizaciones en la díada participan del mismo intercambio y "0" si no participan.

A continuación hemos operacionalizado la innovación en GRH. Al igual que en el caso del tipo de intercambio, se ha creado una matriz de 50x50, según si la tasa de introducción de innovaciones en GRH es baja (la empresa llevo a cabo innovaciones en menos de un $30 \%$ de las prácticas de GRH propuestas), media (hasta un $60 \%$ ) o alta (innovaciones en $61 \%$ o más actividades de GRH) en los dos últimos años. Si bien en términos absolutos pueden considerarse unos niveles altos de innovación, son relativamente característicos de las empresas de alta tecnología. La codificación de cada empresa es " 1 " si ambas organizaciones en la díada comparten intensidad innovadora y " 0 " si tienen diferentes niveles de intensidad innovadora.

\section{Resultados}

Esta sección se organiza en torno a las dos preguntas de investigación propuestas. El primer conjunto de resultados se refiere a las características y propiedades estructurales del intercambio informal de conocimientos GRH. El segundo conjunto examina la relación entre la participación en redes de intercambio y la actividad innovadora de las empresas en materia de GRH. 
Propiedades estructurales de las redes de conocimientos de GRH entre empresas

En primer lugar se presenta la visualización de la red examinada y la posición estructural de las firmas dentro de ella (ver Figura 1 y Figura 2). Los nodos representan las empresas individuales y las flechas ilustran el flujo de conocimiento de GRH.

Figura 1. Red colaborativa de intercambio de conocimiento de GRH (centralidad de grado para tamaño de nodo)

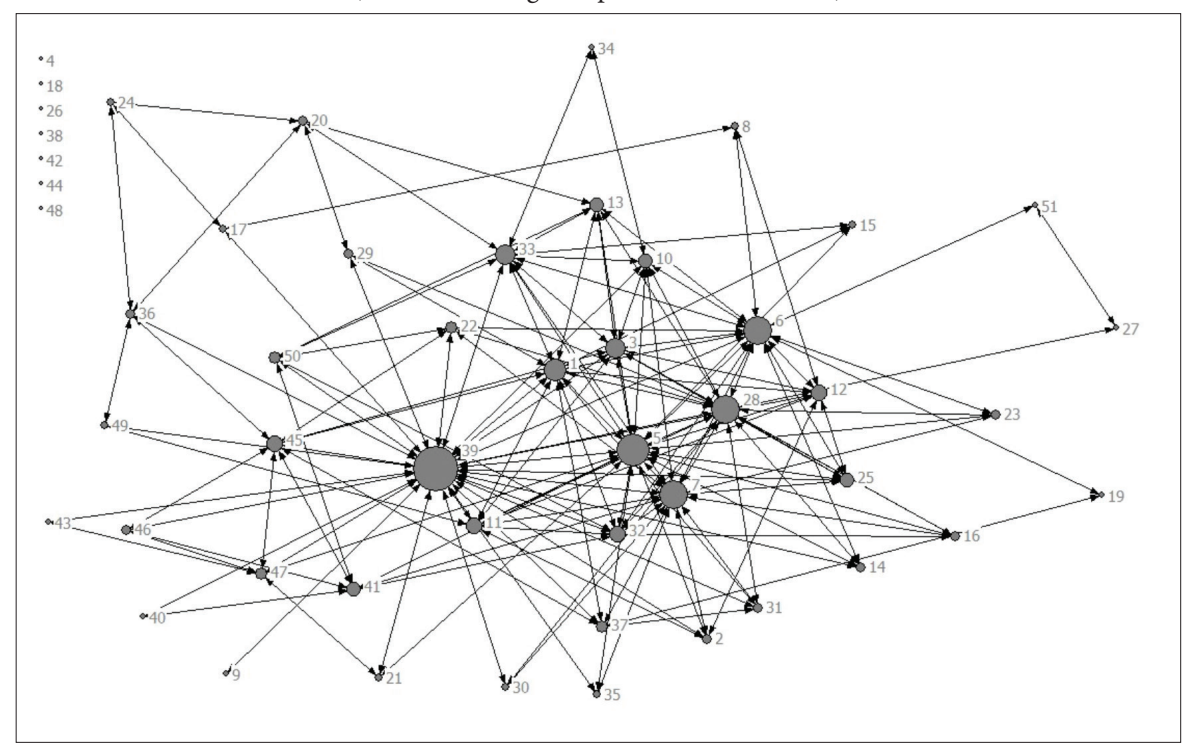

Figura 2. Red formal de intercambio de conocimiento de GRH (centralidad de grado para tamaño de nodo)

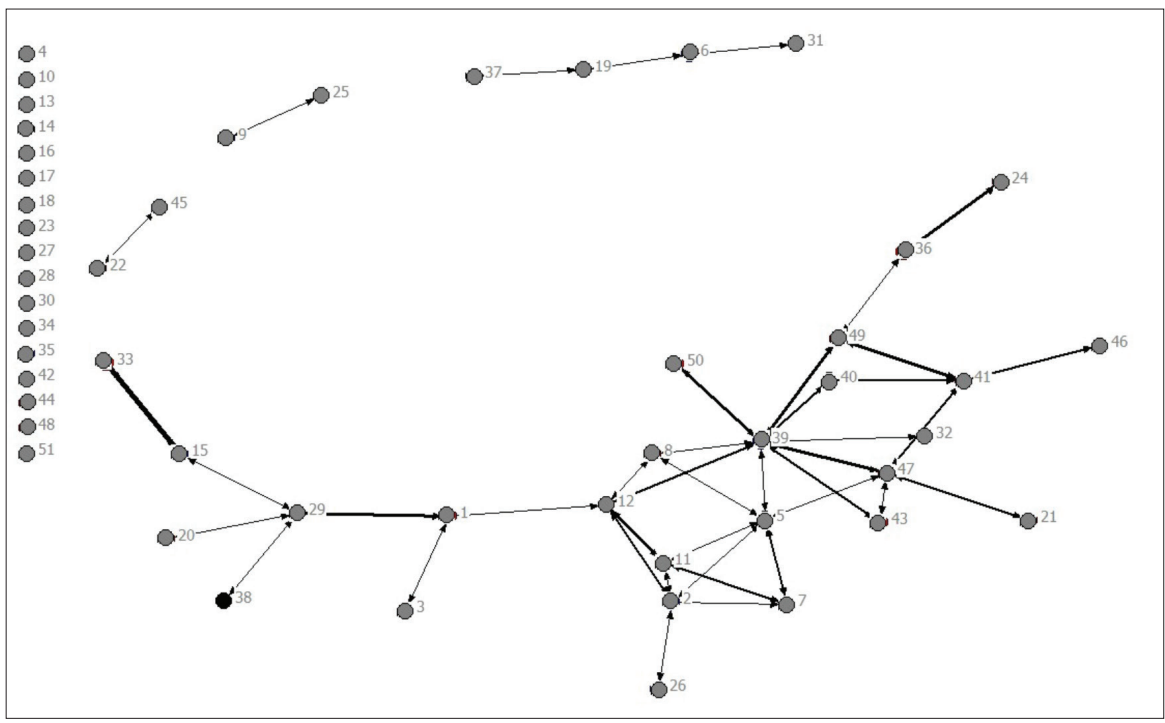

Con respecto a la estructura reticular de las relaciones de intercambio de conocimiento, como se ve en el Gráfico 1, la red revela que las relaciones colaborativas están relativamente consolidadas. Pocas empresas están aisladas del resto de las empresas del parque. La existencia de una red de conocimiento de GRH, posiblemente, puede ser atribuible al hecho de que la mayoría de las empresas en el parque comparten ciertos intereses comunes, al operar en industrias similares (industrias de alta tecnología) y ser similares en tamaño y antigüedad. Sobre la base de nuestro análisis, una observación importante se puede hacer de la red de GRH: las empresas se involucran en relaciones de colaboración que van más allá del intercambio de información sobre aspectos tecnológicos, de mercado o productos (tal y como la literatura ha puesto de manifiesto en diversos estudios) para incluir el conocimiento de GRH. La Figura 2 muestra el sociograma de relaciones formales de intercambio de conocimiento de GRH. En este caso se muestra que determinadas empresas se benefician de la existencia de acuerdos formales con otras organizaciones para intercambiar conocimiento de GRH. Este tipo de intercambios ha sido documentado en la literatura especializada (Martin-Rios, 2014). Por tanto, podemos afirmar que la gran mayoría de las empresas del 
estudio participaron en la red de GRH con el fin de obtener información sobre aspectos relacionados con la GRH, respondiendo a nuestra primera pregunta de investigación.

Varios nodos de coordinación son citados con recurrencia. Estos representan las empresas que se sitúan en el corazón de la red y tienen un gran número de vínculos. En ese sentido, la Oficina de Tecnológica es central en la red, aunque no tenga ningún control de los recursos financieros y de personal de las empresas del parque. Su centralidad podría estar relacionado con su papel como proveedor de conocimiento que desarrolla fuertes lazos con la mayoría de las empresas en la red y sirve como intermediario entre los distintos grupos en el intercambio del conocimiento. Para examinar la distribución de las empresas a través de las redes, se calculó el grado de puntos, es decir, el número de conexiones (las relaciones) que una empresa tiene con otras empresas en cada red de conocimiento. Por ejemplo, la empresa
5 es la principal fuente de información en la red. Esta empresa es una de las más antiguas en el parque, participa activamente en la Asociación de Empresas y es reconocida por su tecnología y la innovación organizacional. Otras empresas como la 2, 3, 11 y 46 son muy activas en la red tanto en la recepción como en la difusión de conocimiento de GRH. Hay una gran variación de actores en lo que respecta a la recepción de información (más que para la difusión de la información). Las empresas $1,7,11$ y 32 son centrales en la recepción de información. Las empresas 7 y 11 son también activas en el intercambio de información y, de alguna manera, actúan como "comunicadores" en la red. En cambio, la empresa 1 recibe abundante información de $\mathrm{GRH}$, pero no transfiere mucha.

A continuación, analizamos los factores los índices de red calculados, incluyendo la densidad, la centralización de la red y la reciprocidad (ver Tabla 2).

Tabla 2. Resultados estructurales de la red

\begin{tabular}{|c|c|c|c|c|c|}
\hline & Tamaño* & Nodos aislados & Índice centralización (\%) & Densidad & Lazos recíprocos (\%) \\
\hline $\begin{array}{l}\text { Red formal } \\
\text { (transaccional) }\end{array}$ & 51 & $17 / 51=33.3 \%$ & 33.35 & 0.05 & $28.72 \%$ \\
\hline
\end{tabular}

* Incluyendo la Oficina de Transferencia

Respecto a la centralización de la red, cuanto más centralizada es la red mayor es el número de empresas en una posición que les permite controlar el flujo de conocimientos. La estructura de ambas redes de conocimiento de GRH es relativamente descentralizada: 38,7\% para la red formal (transaccional) y $33 \%$ para la red informal (colaborativa). Para calcular la densidad de los flujos de conocimiento entre empresas, se calculó el porcentaje de densidad de cada red. Para la red informal, la medida de densidad tiene el valor de 0,07 y para la red formal 0,05. En base a estos valores, se podría especular que el flujo de conocimiento de baja densidad descansa en una serie de contactos relativamente poco frecuentes y poco estructurados entre un número considerable de empresas. Respecto a las propiedades relacionales (naturaleza de los vínculos) de la red de conocimiento de GRH, el porcentaje de relaciones recíprocas es $21,54 \%$ y $29 \%$. De todas las parejas de actores que tienen alguna conexión, el 53\% de las parejas tienen una conexión recíproca. Esto podría sugerir una estructuración más bien no-jerárquica de la red de GRH.

\section{La relación entre la red de conocimiento de GRH y la innovación en GRH}

La segunda pregunta de investigación propuesta investiga la relación entre la participación de las empresas en redes de intercambio de conocimiento de GRH y la capacidad de innovación en materia de GRH. La literatura en relaciones inter-organizativas muestra la existencia de una relación positiva entre ambos fenómenos en aspectos tales como la innovación tecnológica, la innovación de producto, o la innovación social. Hay, sin embargo, una carencia de estudios que permitan aseverar que la participación en redes de conocimiento de GRH tiene un efecto positivo en la actividad innovadora en GRH.
En este estudio las empresas se clasificaron en función de la mayor o menor intensidad de sus innovaciones en GRH entre las que tenían baja intensidad de innovaciones (menos de un 30\%), media (hasta un $60 \%$ ), y alta intensidad con más de un $61 \%$ de innovaciones en GRH en los dos últimos años. La variable resultante está representada gráficamente en la Figura 3 donde se observa que la mayoría de empresas presentan unos niveles moderados de innovación en GRH.

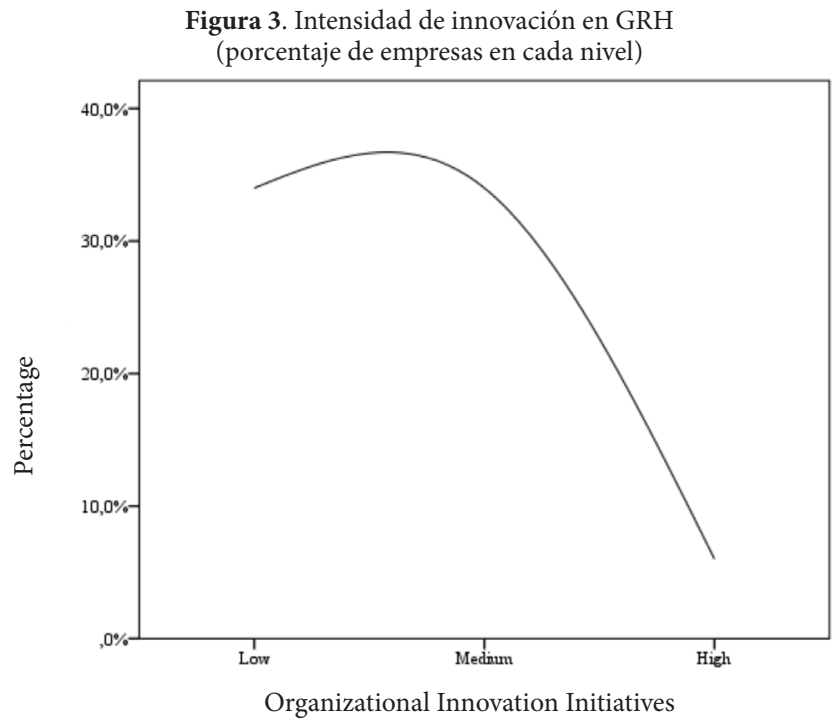

El análisis de varianza ANOVA permite observar diversas relaciones estadísticamente significativas entre la actividad innovadora y la participación en redes de intercambio (Tabla 3). Por un lado, hay una 
relación sólida (sig.+.01) entre la intensidad innovadora alta y la participación en redes tanto formales como informales de intercambio de conocimiento de GRH. Las empresas más innovadoras juegan un papel muy activo en las dinámicas de intercambio inter-organizacionales. Asimismo, se observa una relación positiva (sig.+.05) entre las empresas de intensidad innovadora media y la participación en redes cooperativas o informales de intercambio. Este tipo de empresas optan por obtener conocimiento de GRH a través de relaciones colaborativas más que a través de redes contractuales. Finalmente, hay una relación negativa y significativa entre una intensidad innovadora baja y la participación en redes formales de conocimiento (sig.+.05). Respecto a las redes informales la relación no es estadísticamente significativa. Así, la dirección apunta a que las empresas con menor intensidad innovadora participan poco tanto en redes formales como en redes informales.

Tabla 3. Actividad innovadora y participación en redes de conocimiento (ANOVA).

\begin{tabular}{llll}
\hline \multirow{2}{*}{$\begin{array}{l}\text { Participación } \\
\text { en redes }\end{array}$} & \multicolumn{3}{l}{ Intensidad innovadora de la empresa } \\
\cline { 2 - 4 } & $\begin{array}{l}\text { Baja innovación } \\
\text { GRH }\end{array}$ & $\begin{array}{l}\text { Media innovación } \\
\text { GRH }\end{array}$ & $\begin{array}{l}\text { Alta innovación } \\
\text { GRH }\end{array}$ \\
\hline $\begin{array}{l}\text { Intercambio } \\
\text { formal }\end{array}$ & $(-)^{*}$ & $(-)$ & $(++)^{* *}$ \\
\hline $\begin{array}{l}\text { Intercambio } \\
\text { informal }\end{array}$ & $(-)$ & $(+)^{*}$ & $(++)^{* *}$ \\
${ }^{*}=$ Sig. 0.05 & & \\
$* *=$ Sig. 0.01 & & &
\end{tabular}

\section{Discusión}

El propósito de este trabajo es analizar los factores que determinan el intercambio de conocimiento de GRH y su relación con los niveles de innovación de un grupo de empresas localizadas en un parque científico y tecnológico en España. La principal conclusión alcanzada es que las empresas, a través de sus gerentes y el personal clave en materia de gestión de personas, son conocedoras de la importancia de incorporar conocimiento a partir de fuentes externas; puesto que dicha participación tiene una relación positiva con la introducción de innovaciones en GRH. Esta investigación está guiada por una concepción teórica del intercambio de conocimiento de GRH como un proceso dinámico profundamente enraizado en un sistema complejo de redes de relaciones, donde el establecimiento y el mantenimiento de lazos inter-organizativos tanto formales como informales estimulan el desarrollo de tales intercambios (Sammarra y Biggiero, 2008). Los flujos de conocimiento tienen lugar a través de estos intercambios con los posibles efectos beneficiosos en forma de un mayor esfuerzo innovador en GRH por parte de las empresas participantes y, en última estancia, la formación de ventajas competitivas.

Nuestros resultados ofrecen pruebas preliminares sobre la función positiva que las redes entre organizaciones desempeñan en la difusión de conocimientos relacionados con la GRH. En respuesta a nuestra pregunta de investigación, en qué medida se producen intercambios de conocimiento de GRH entre empresas independientes, nuestra conclusión es positiva. Nuestros hallazgos muestran que la red de conocimiento de GRH empresa a empresa estaba relativamente bien consolidada. En ese sentido, podemos concluir que las empresas objeto de este estudio participan activamente en la difusión y el intercambio de conocimientos de GRH con otras empresas. Los resultados de los análisis sociométricos indican que las empresas ubicadas en el PCT participan en dichas redes con el propósito de obtener acceso a las capacidades de GRH en manos de otras empresas. Como resultado, la red de conocimientos de GRH era relativamente descentralizada y tenía baja densidad, sobre todo es interactiva (en función de la proporción relativamente alta de relaciones recíprocas) dado que la mayoría de las empresas son accesibles a todas las demás. Todo lo cual parece favorecer el intercambio de una cantidad considerable de conocimientos GRH.

Además, el estudio plantea una posible relación positiva entre la participación en redes de conocimiento y la actividad innovadora en GRH. En este sentido, la literatura existente da indicaciones de la posible relación (Martin-Rios, 2014); sin embargo, la carencia de estudios específicos no permiten alcanzar conclusiones respecto a la GRH. En concreto, este estudio apunta a la importancia que las empresas otorgan a adquirir nuevos conocimientos de GRH como uno de los principales motivadores y beneficios para llevar a cabo innovaciones de GRH. Puesto que las competencias básicas de la empresa moderna están ineludiblemente ligadas al conocimiento (Grant, 1996; MartinRios y Erhardt, 2017), la cultura innovadora (Erhardt, Martin-Rios y Heckscher, 2016; Erhardt, Martin-Rios y Way, 2009; Parga, MartinRios y Criado, 2013) y los sistemas de innovación tecnológica y no tecnológica (Martin-Rios y Pasamar, 2017; Martin-Rios, Pasamar y Gonzalez-Perez, 2016), los flujos de conocimiento son indispensables para promover el aprendizaje organizacional (Martin-Rios y Erhardt, 2017; Septiem y Martin-Rios, 2011). Lo cual permite a la empresa ampliar, mejorar y modificar sus stocks de conocimientos. Y, en definitiva, favorecer el desarrollo de las capacidades dinámicas de la empresa (Teece et al., 1997). En ese sentido, esta conclusión se suma a un debate central en la GRH como fuente de creación de valor (Delery y Doty, 1996), mediante el cual el conocimiento de GRH ofrece oportunidades de aprendizaje e innovación para las empresas (Martin-Rios, 2014). El aprendizaje organizacional es una capacidad fundamental de la organización que se ha planteado como una importante fuente de ventaja competitiva sostenible (Fiol y Lyles, 1985). Las redes entre organizaciones devienen un aspecto destacado en este proceso de aprendizaje (Martin-Rios y Erhardt, 2017). De ello se desprende, que la participación en redes de conocimientos de GRH pueden ser un medio para crear y fomentar una ventaja competitiva.

Una implicación práctica derivada de este estudio radica en la oportunidad que supone para los profesionales de GRH el fomentar el establecimiento de lazos colaborativos con personas externas a la propia organización. De este modo, pueden establecerse lazos informales relacionales, los cuales implican no solo cierta legitimidad, sino también un cierto grado de reciprocidad (Szulanski, 1996). Aprender cómo se propaga la GRH de conocimiento entre las empresas puede dar lugar a formas más eficaces de aprovechar esas corrientes, que pueden contribuir al aumento de las existencias de conocimiento de GRH. Además, el intercambio de las experiencias cooperativas tiene 
consecuencias positivas, no sólo para las organizaciones que han obtenido los conocimientos de GRH, sino también para aquellos que comparten información, que pueden lograr una mayor legitimidad y prestigio. Dicha aseveración enlaza con estudios anteriores en la materia. Por ejemplo, Kossek (1987) menciona en su estudio la legitimidad y el prestigio como razones para participar en redes interorganizacionales de las cuales los ejecutivos de la firma son miembros para intercambiar información sobre las tendencias actuales de GRH. Asimismo, Williamson y Cable (2003) sugieren que la legitimidad juega papel preponderante en el establecimiento de relaciones de intercambio: quienes toman las decisiones en las empresas imitan las prácticas que ellos consideran legítimas, dado que han producido resultados positivos para dichas empresas.

Cabe señalar por ultimo diversas limitaciones del estudio. El método que se emplea para evaluar empíricamente dicha relación es el análisis de la varianza ANOVA, el cual posee diversas limitaciones relacionadas con la necesidad de realizar un muestreo aleatorio. Futuras investigaciones deberían utilizar modelos de análisis que faciliten la robustez de los resultados obtenidos. Otros vacíos identificados por los autores constituyen oportunidades de investigación. Por ejemplo, las redes de conocimiento son vitales para las organizaciones como fuente de conocimiento y, por tanto, como un elemento esencial que aporta valor a los objetivos estratégicos de la empresa. Se debería profundizar sobre las motivaciones de los actores implicados en el intercambio de conocimiento para su participación o no en redes colaborativas y las ganancias y perjuicios que se pueden derivar de dicha participación o de su ausencia. Mientras tanto surgen nuevas oportunidades innovadoras alentadas por el intercambio de conocimiento lo que podría indicar que las empresas que tratan de adaptarse a estas transformaciones son capaces de perseverar. Estos aspectos merecen ser considerados en el desarrollo de futuros estudios.

En conclusión, este estudio proporciona un puente entre los teóricos de redes sociales y los teóricos de la GRH al analizar las características y propiedades de los canales de intercambio, a través de los cuales, el conocimiento de GRH se propaga. Si las redes entre empresas son cruciales para el flujo de conocimiento y, por tanto, para el aprendizaje organizacional y la competitividad, este estudio se centra en analizar cuál es la relación existente entre la participación en relaciones de colaboración para aumentar el acceso a los conocimientos GRH y la implementación de innovaciones en GRH. Actualmente sabemos relativamente poco sobre las prácticas de éxito en la gestión del intercambio de conocimientos en la GRH y acerca de las relaciones diádicas entre las empresas que potencialmente mejoran el nivel de colaboración que se necesita para impulsar el intercambio de conocimientos de GRH. Hemos concluido que las empresas participan en tales flujos de conocimiento dada una posible ganancia instrumental: la mejora de sus stocks de conocimiento manteniendo estables los costes de transacción; así como ciertas ganancias relacionales: el establecimiento de relaciones de reciprocidad y la ganancia de legitimidad y prestigio en su entorno.

\section{Breve nota biográfica de los autores}

Manuel Septiem is adjunct professor of Economics of Enterprise at the Universitat de les Illes Balears (Spain) and of Management at Universidad Autonoma de Madrid (Spain). Currently, he works toward his doctoral dissertation in the field of human resource management. His research interest are concerned with inter-firm collaborations and knowledge exchanges with an emphasis on people management in knowledge-intensive work settings.

Carlos Martin-Rios ( $\mathrm{PhD}$, Rutgers University) is associate professor of innovation at the Ecole hôtelière de Lausanne // HES-SO University of Applied Sciences Western Switzerland, and Managing Director of the Research Center Hospitality Innovation Network (HIN-EHL). He performs research that aims to understand and predict the factors that determine non-technological innovation (e.g., business model innovation, organizational innovation, and management innovation). He is the author or co-author of over 40 refereed journal publications, including International Small Business Journal, Creativity and Innovation Management, Journal of Business Research, and R\&D Management.

\section{Bibliografía}

Appleyard, M. (1996). How does knowledge flow? Inter-firm patterns in the semiconductor industry. Strategic Management Journal, 17, 137-154.

Barney, J.B. (1991). Firm resources and sustained competitive advantage. Journal of Management, 17, 99-120.

Becker, M.H. (1970). Sociometric Location and Innovativeness: Reformulation and Extension of the Diffusion Model. American Sociological Review, 35(2), 267-282.

Björkman, I., \& Lervik, J. (2007). Transferring HR practices within multinational corporations. Human Resource Management Journal, 17(4), 320-335.

Borgatti, S. (2007). NetDraw network visualization software. Harvard: Analytic Technologies.

Borgatti, S. P., \& Everett, M. G. (1997). Network analysis of 2-mode data. Social Networks, 19(3), 243-269.

Borgatti, S. P., \& Cross, R. (2003). A relational view of information seeking and learning in social networks. Management Science, 49(4), 432-445.

Borgatti, S., Everet, M., \& Freeman, L. (2002). UCINET for Windows: Software for social network analysis. Harvard: Analytic Technologies.

Brass, D. (1995). A social network perspective on human resources management. Research in Personnel and Human Resources Management, 13, 39-79.

Breschi, S., \& Lissoni, F. (2001). Knowledge spillovers and local innovation systems: a critical survey. Industrial and Corporate Change, 10(4), 975-1005. 
Burns, L.R., \& Wholey, D.R. (1993). Adoption and abandonment of matrix management programs: Effects of organizational characteristics and interorganizational networks. Academy of Management Journal, 36, 106-138.

Burt, R. S., Minor, M. J., \& Associates (Eds.) (1983) Applied network analysis: A methodological introduction. Beverley Hills, CA: Sage.

Castells, M. \& Hall, P. (1994). Technopoles of the world: The making of Twenty-First-century industrial complexes. London: Routledge.

Coleman, J. (1958). Relational Analysis: The study of social organizations with survey methods. Human Organization, 17(4), 28-36.

Coleman, J.S., Katz, E. \& Menzel, H. (1966). Medical innovation: A diffusion study. Indianapolis: Bobbs-Merrill.

Cross R., Nohria N., \& Parker A. (2012). Six myths about informal networks-and how to overcome them. Sloan Management Review, 43.

Delery, J.E., \& Doty, D. H. (1996). Modes of theorizing in strategic human resource management: Tests of universalistic, contingency, and configurational performance predictions. Academy of Management Journal, 39, 802-835.

Dierickx, I., \& Cool, K. (1989). Asset stock accumulation and sustainability of competitive advantage. Management Science, 35(12), 1504-1513.

Diez-Vial, I and Montoro-Sanchez A (2016) How knowledge links with universities may foster innovation: The case of a science park. Technovation, 50: 41-52.

Dyer, J. H., \& Nobeoka, K. (2000). Creating and managing a highperformance knowledge sharing network: The Toyota case. Strategic Management Journal, 21, 345-367.

Easterby-Smith, M., Lyles, M., \& Tsang, E.W. (2008). Inter-organizational knowledge transfer: Current themes and future prospects. Journal of Management Studies, 45(4), 677-690.

Erhardt, N., Martin-Rios, C. \& Way, S. (2009). From bureaucratic forms towards team-based knowledge work systems: Implications for human resource management. International Journal of Collaborative Enterprise, 1(2): 160-179

Erhardt, N., Martin-Rios, C. \& Heckscher, C. (2016). Am I doing the right thing? Rituals as institutional learning mechanisms for organizational culture in restaurants. International Journal of Hospitality Management, 59: 31-41

Fiol, C. M., \& Lyles, M. A. (1985). Organizational learning. Academy of Management Review, 10, 803-813.

Fligstein, N. (1985). The spread of the multidivisional form among large firms, 1919-1979. American Sociological Review, 50(3), 377391.
Freeman, L.C. (1979). Centrality in social networks: Conceptual clarification. Social Networks, 1, 215-239.

Friedman, R.A., \& Podolny, J. (1992). Differentiation of boundary spanning roles: Labor negotiations and implications for role conflict. Administrative Science Quarterly, 37, 28-47.

Granovetter, M.S. (1979). The theory gap in social network analysis. In P.W. Holland \& S. Leinhardt (Eds.), Perspectives on social network research (pp. 501-518). New York: Academic.

Granovetter, M.S. (1985). Economic action and social structure: The problem of embeddedness. American Journal of Sociology, 91: 481-510.

Huselid, M.A. (1995). The impact of human resource management practices on turnover, productivity, and corporate financial performance. Academy of Management Journal, 38, 635-72.

Hayter CS (2013) Conceptualizing knowledge-based entrepreneurship networks: Perspectives from the literature. Small Business Economics 41, 899-911.

Kilduff, M., \& Brass, D. J. (2010). Organizational social network research: Core ideas and key debates. Academy of Management Annals, 4(1), 317-357.

Kossek, E. E. (1987). Human resources management innovation. Human Resource Management, 26, 71-92.

Kumar, N., Stern, L. W., \& Anderson, J. C. (1993). Conducting interorganizational research using key informants. Academy of Management Journal, 36, 1633-1651.

Lengnick-Hall, C.A., \& Lengnick-Hall, M.L. (2003). HR's role in building relationship networks. Academy of Management Executive, 17, 53-66.

Lepak, D.P., \& Snell S.A. (2002). Examining the human resource architecture: The relationships among human capital, employment, and human resource configurations. Journal of Management, 28(4), 517-543.

Martin-Rios, C. (2014). Why do firms seek to share human resource management knowledge? The importance of inter-firm networks. Journal of Business Research, 67(2), 190-199.

Martin-Rios, C. \& Erhardt, N. L. (2008). Organizational knowledge transfer through human resource management: international diffusion of managerial performance management. European Journal of International Management, 2: 170-191.

Martin-Rios, C. \& Erhardt, N. (2017). Small business activity and knowledge exchange in informal interfirm networks. International Small Business Journal, 35(3): 285-305.

Martin-Rios, C. \& Parga, E. (2016). Service response to economic decline: Management innovation as enabler of organizational renewal. Journal of Business Research, 69(8): 2890-2900 
Martin-Rios, C. \& Pasamar, S. (2017). Service innovation in times of economic crisis: The strategic adaptation activities of the top EU service firms. R\&D Management. doi:10.1111/radm.12276

Martin-Rios, C. \& Septiem, M. (2013). Cooperación e intercambio de conocimiento en redes inter-organizativas informales. REDES-Revista Hispana para el Análisis de Redes Sociales, 24(1): 193-216.

Martin-Rios, C., Pasamar, S., \& Gonzalez-Perez, C. (2016) Innovación organizativa en organismos de investigación. Dirección y Organización, 59: 4-17

Martin-Rios, C., Pougnet, S., \& Nogareda, A.M. (2017). Teaching HRM in contemporary hospitality management: A case study drawing on HR analytics and big data analysis. Journal of Teaching in Travel and Tourism, 1: 34-54

Mazza, C., \& Alvarez, J.L. (2000). Haute couture and prêt-à-porter: The popular press and the diffusion of management practices. Organization Studies, 21, 567-588.

Moreno, J.L. (1934) Who Shall Survive? A new approach to the problem of human interrelations. Washington, DC: Nervous and Mental Disease Publishing Co.

Paauwe, J., \& Boselie, P. (2005). Best practices ... in spite of performance: Just a matter of imitation? International Journal of Human Resource Management, 16 (6), 987-1003.

Parga, E., Martin-Rios, C., \& Criado, F. (2013). Organizational and management innovation as a driving force of business renewal. Journal of Technology Management \& Innovation, 8(2): 132-143

Parmigiani, A. \& Rivera-Santos, M. (2011). Clearing a path through the forest: A meta-review of interorganizational relationships. Journal of Management, 37(4), 1108-1136.

Powell, W., Koput, K., \& Smith-Doerr, L. (1996). “Interorganizational collaboration and the locus of innovation: Networks of learning in biotechnology". Administrative Science Quarterly, 41: 116-145.

Powell, W.W., \& Grodal, S. (2005). Networks of Innovators. En J. Fagerberg, D. Mowery, y R.R. The Oxford Handbook of Innovation, Oxford University Press, pp. 56-85

Powell, W.W., Koput, K.W., \& Smith-Doerr, L. (1996). Inter-organizational collaboration and the locus of innovation: networks of learning in biotechnology. Administrative Science Quarterly, 41, 116-145.

Reagans, R., \& McEvily, B. (2003). Network structure and knowledge transfer: The effects of cohesion and range. Administrative Science Quarterly, 48(2), 240-267
Rubinstein, S., Martin-Rios, C., Erhardt, N., Hoffer-Gittell, J., \& George V.P. (2016). Organizational responses to uncertainty in the airline industry: Changes in patterns of communication networks. Journal of Air Transport Management, 57: 217-227

Sammarra, A., \& Biggiero, L. (2008). Heterogeneity and specificity of inter-firm knowledge flows in innovation networks. Journal of Management Studies, 45: 800-829.

Schuler, R., Jackson, S., \& Luo, Y. (2004). Managing human resources in cross-border alliances. London, Routledge.

Scott, J. (1991). Social network analysis: A handbook. Thousand Oaks, CA: Sage.

Scott, R. (2001). Institutions and organizations. Thousand Oaks, CA: Sag Publications.

Septiem, M. \& Martin-Rios, C. (2011). La existencia de ventaja competitiva en la difusión de innovaciones en la gestión de recursos humanos: El impacto de las redes de conocimiento inter-organizacional Boletín de Estudios Económicos, 66(203):199-218

Szulanski, G. (1996). Exploring internal stickiness: Impediments to the transfer of best-practice within the firm. Strategic Management Journal, 17: 27-43.

Teece, D.J., Pisano, G., \& Shuen, A. (1997). Dynamic capabilities and strategic management. Strategic Management Journal, 18(8), 509-533.

Uzzi, B. (1996). The sources and consequences of embeddedness for the economic performance of organizations: The network effect. American Sociological Review, 61(4), 674-698.

Wasserman, S., \& Faust, K. (1994). Social network analysis: Methods and applications. New York: Cambridge University Press.

Wellman, B. (1983). Network analysis: Some basic principles. Sociological Theory, 1: 155-200.

Westphal, J.D., \& Zajac, E.J. (1997). Defections from the inner circle: Social exchange, reciprocity, and the diffusion of board independence in U.S. corporations. Administrative Science Quarterly, 42, 161-183.

Williamson, I.O., \& Cable, D.M. (2003). Organizational hiring partners, interfirm network ties, and interorganizational imitation. Academy of Management Journal, 46 (3), 349-358.

Wright, P. M., Dunford, B. B., \& Snell, S. A. (2001). Human resources and the resource based view of the firm. Journal of Management, 27(6), 701 . 
